\title{
Particle acceleration and the origin of the very high energy emission around black holes and relativistic jets
}

\author{
Elisabete M. de Gouveia Dal Pino ${ }^{1}$, Grzegorz Kowal ${ }^{2}$, Luis \\ Kadowaki $^{1}$, Tania E. Medina-Torrejón ${ }^{1}$, Yosuke Mizuno ${ }^{3}$ and \\ Chandra Singh ${ }^{4}$

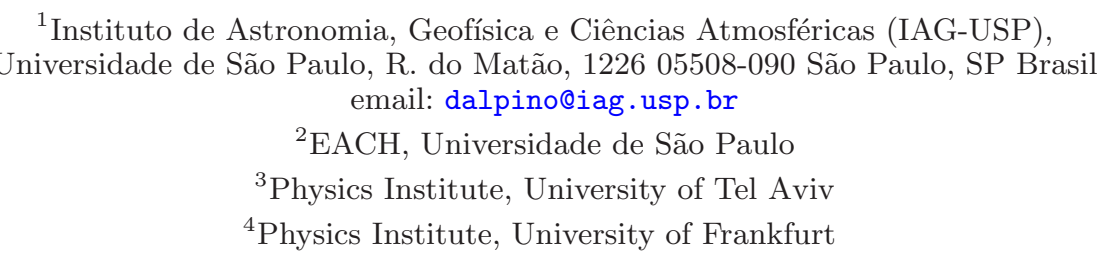

\begin{abstract}
Particle acceleration induced by fast magnetic reconnection may help to solve current puzzles related to the interpretation of the very high energy (VHE) and neutrino emissions from AGNs and compact sources in general. Our general relativistic-MHD simulations of accretion disk-corona systems reveal the growth of turbulence driven by MHD instabilities that lead to the development of fast magnetic reconnection in the corona. In addition, our simulations of relativistic MHD jets reveal the formation of several sites of fast reconnection induced by current-driven kink turbulence. The injection of thousands of test particles in these regions causes acceleration up to energies of several PeVs, thus demonstrating the ability of this process to accelerate particles and produce VHE and neutrino emission, specially in blazars. Finally, we discuss how reconnection can also explain the observed VHE luminosity-black hole mass correlation, involving hundreds of non-blazar sources like Perseus A, and black hole binaries.
\end{abstract}

Keywords. acceleration of particles, black hole physics, galaxies: active, MHD

\section{Introduction}

Since de Gouveia Dal Pino \& Lazarian (2005) proposed for the first time that particles could be accelerated in a first-order Fermi process within fast reconnection sites (current sheets), considerable progress has been attained in this field mainly boosted by numerical modeling employing both PIC (e.g. Drake et al. 2006, Zenitani \& Hoshino 2008, Lyubarsky et al. 2008, Clausen-Brown et al. 2012, Cerutti et al. 2014, Sironi et al. 2014, Guo et al. 2016) and test particle-plus-MHD techniques (e.g., Kowal, de Gouveia Dal Pino \& Lazarian 2011, 2012, del Valle, de Gouveia Dal Pino \& Kowal 2016). These studies have successfully tested the theoretical predictions identifying an exponential growth of the particles energy with time while trapped within current sheets (e.g., Kowal et al. 2011, 2012), and a power-law energy spectrum for the accelerated particles with spectral indices $\sim-1$ to -2 (e.g. Kowal et al. 2012, del Valle et al. 2016), and a power-law dependence of the acceleration rate with the particle energy (with powerlaw indices $\sim 0.3-0.6$ for a non-relativistic background (del Valle et al. 2016). These findings are general and in principle applicable to any system, specially in turbulent 
magnetically dominated regions. $\nmid$ This mechanism is now regarded as important also beyond the solar system (where it has been extensively investigated). In particular, it may explain current puzzles related to very high energy phenomena observed in compact sources like AGNs, black hole X-ray binary systems, and GRBs. In this talk, we discuss this process in the framework of these sources, i.e., in the surrounds of black holes (BHs) and relativistic jets (see also de Gouveia Dal Pino et al. 2015, 2016 for recent reviews).

\section{Magnetic Reconnection Acceleration in Relativistic Jets}

AGNs with highly beamed jets pointing to the line of sight, namely blazars, are the most common sources of $\gamma$-rays. This emission is generally attributed to diffusive shock particle acceleration along the jet which is strongly Doppler boosted producing apparently very high uxes. However, in some cases (e.g., PKS2155-304 source; Aharonian et al. 2007 ), observed very high variability, of the order of minutes in the $\mathrm{TeV}$ range, implies extremely compact and fast acceleration/emission regions $\left(<R_{S} / c\right)$ with Lorentz factors much larger than the typical bulk values $(\Gamma \sim 1-10)$ expected for such sources, in order to avoid electron-positron pair creation. So far, the only model able to explain this high variability at $\mathrm{TeV}$ emission seems to be magnetic reconnection involving misaligned current sheets inside the jet (e.g. Giannios et al. 2009; see also Kushwaha et al. 2017). Fast reconnection acceleration has been also invoked to explain the transition from magnetically to kinetically dominated flow and the prompt gamma-ray emission in GRBs (Zhang \& Yan 2011).

To probe this process, we have recently performed 3D relativistic MHD simulations of rotating Poyinting flux dominated tower jets with initial helical fields (Singh, Mizuno \& de Gouveia Dal Pino 2016). Considering models with a ratio between the magnetic and the rest mass energy of the flow $\sigma=1$, and different density ratios between the jet and the environment, we induced precession perturbations that quickly developed currentdriven kink (CDK) modes. Figure 1 (left) shows an example of a jet with density larger than that of the environment. The results evidence the propagation of a helical kinked structure along the jet that causes substantial dissipation of magnetic into kinetic energy. We also identify regions of maximum current density (labeled with black points) that trace filamentary current-sheets, where turbulent fast magnetic reconnection driven by the CDK instability takes place with rates $\sim 0.05 V_{A}$ (Singh et al. 2016; see also Kadowaki et al., in prep.). The right diagram of Figure 1 depicts the kinetic energy evolution of a distribution of 10,000 particles that were accelerated in situ within the reconnection regions of the relativistic jet depicted in the left panel. Starting with energies $\operatorname{sim} 1 \mathrm{MeV}$, the particles undergo an exponential acceleration once they are trapped in the reconnection sheets (as in Kowal et al. 2012, del Valle et al. 2017), until a saturation level, when then the accelerated particles Larmor radius become larger than the acceleration regions. The initial background magnetic field in this simulation had a maximum value $B=0.13$ $\mathrm{G}$ at the jet axis, but we have also considered simulations with values up to 100 times larger. In this case, when the particles enter the exponential regime, they are accelerated up to energies $\sim 10^{19} \mathrm{eV}$ at sub-pc distances within an AGN jet (Medina-Torrejon, de Gouveia Dal Pino, Kowal, Mizuno, Singh, Kadowaki, in prep.). The implications of these results are very important for relativistic jets in general, as they indicate that these accelerated protons can produce $\gamma$-rays in very compact regions and also neutrinos, as recently detected in the blazar TXS 0506+056 (IceCube collaboration et al. 2018).

$\dagger$ The presence of turbulence makes magnetic reconnection naturally very fast, with reconnection velocities being a substantial fraction of the local Alfven speed (Lazarian \& Vishniac 1999, Kowal et al. 2009). 

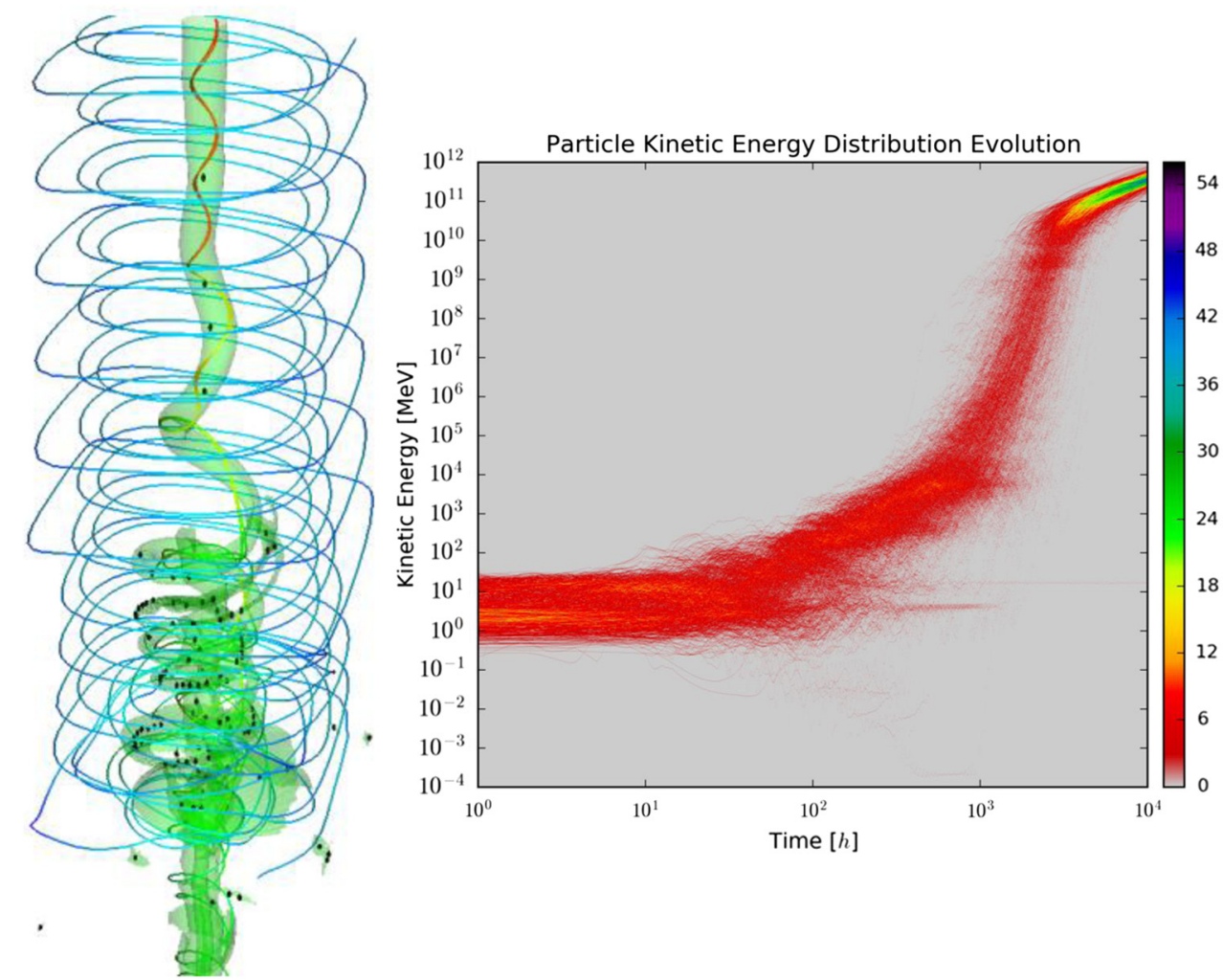

Figure 1. Current-driven kink instability and fast magnetic reconnection in a relativistic MHD jet. The left diagram depicts 3D density isosurfaces (green) with superposed magnetic field lines (black) (extracted from Singh et al. 2016). The black points identify the sites of fast reconnection which were detected employing the technique described in Kadowaki, de Gouveia Dal Pino \& Stone (2018; see also Kadowaki et al., in prep.). Right diagram shows the time evolution of the kinetic energy of 10,000 test particles (protons) that were injected with an initial Maxwelian velocity distribution in the relativistic jet of the left diagram (with initial maximum background magnetic field scaled to $B=0.13 G$ ). Once particles are trapped within reconnection regions they are exponentially accelerated up to energies $\sim 10^{17} \mathrm{eV}$ in this model. Simulations performed with magnetic field one-hundred times larger, allow for particle acceleration up to $10^{19} \mathrm{eV}$ (Medina-Torrejon, de Gouveia Dal Pino, Kowal, Mizuno, Singh, Kadowaki, in prep.).

\section{Magnetic reconnection acceleration around black holes}

Recently, a few non-blazar sources which belong to the branch of low luminosity AGNs (or simply LLAGNs), have been also detected at TeV energies by ground based gammaray observatories (e.g., the radio galaxies Per A, M87, Cen A, and IC 310; Sol et al. 2013; see also E. Torresi these Procs.). The angular resolution and sensitivity of these detectors is still so poor that it is hard to establish if it comes from the jet or from the core. These very high energy (VHE) detections were surprising because, besides being highly underluminous, the viewing angle of the jets of these sources is of several degrees, therefore allowing for only moderate Doppler boosting. These characteristics make it hard to explain the VHE of these sources adopting the same standard scenario as in blazars. Furthermore, observations of short time scale variability in the $\gamma$-ray emission of these sources indicate that it is produced in a very compact region that could be, perhaps, the core. These findings led to the search for alternative particle acceleration 


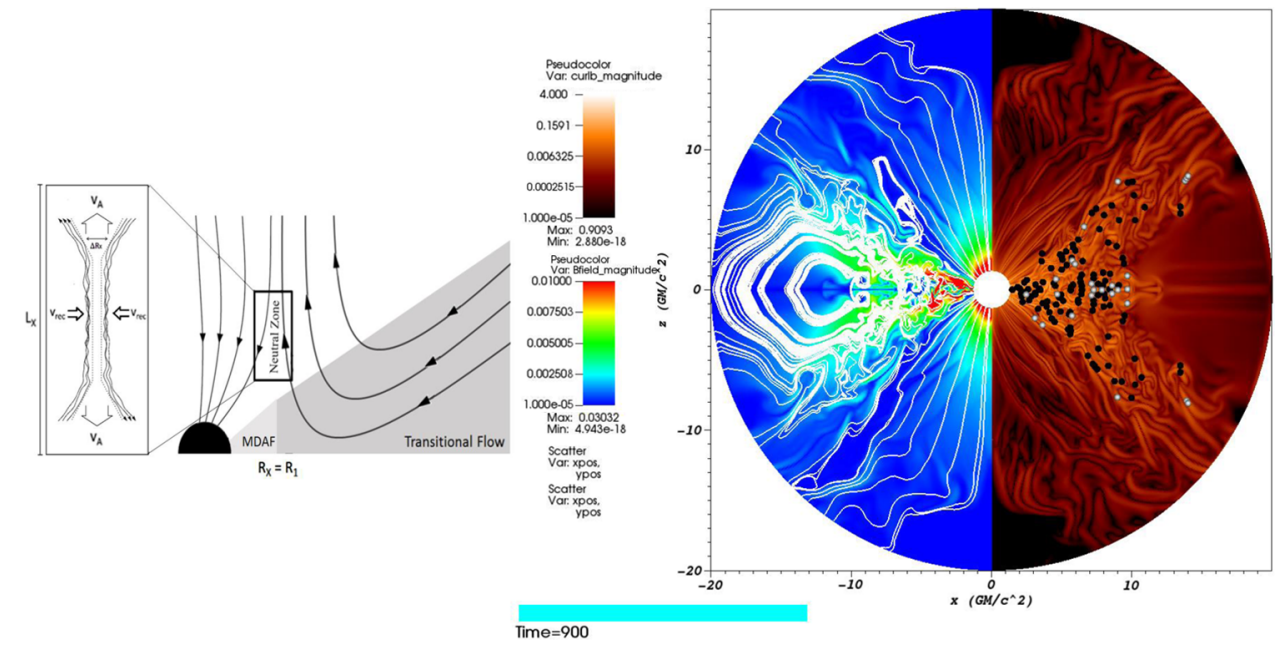

Figure 2. Left: Schematic representation of magnetic reconnection between the lines of the BH magnetosphere and those emerging from the accretion disk into the corona. Reconnection is fast due to the presence of turbulence in the reconnection zone (as indicated in the detail) and allows for extraction of large amounts of reconnection power. Particle acceleration may also naturally occur in the magnetic reconnection zone by Fermi process (adapted from Singh et al. 2015). This representation is suitable for a magnetized ADAF type accretion disk, but we obtain similar results when considering a geometrically thin accretion disk (de Gouveia Dal Pino \& Lazarian 2005, Kadowaki et al. 2015). Right: 2D general relativistic MHD (GR-MHD) simulation of a torus accretion flow around a BH evidencing the development of turbulent reconnection induced by the MRI instability in the magnetic field and current-density distributions. The black and white dots in the current-density plot identify the sites of fast reconnection using the technique described in Kadowaki, de Gouveia Dal Pino \& Stone (2018).

scenarios involving the production of the VHE in the core region of these sources (e.g. de Gouveia Dal Pino et al. 2010; 2016 and references therein).

Another striking evidence was found by Kadowaki, de Gouveia Dal Pino \& Singh (2015) who plotted in the same diagram the $\gamma$-ray luminosity versus the BH mass for more than 230 sources including non-blazar LLAGNs, black hole X-ray binaries (BHBs), blazars and GRBs, spanning over $\sim 10$ orders of magnitude in mass and power. This diagram reveals two distinct branches, or correlation trends. One that is followed by blazars and GRBs, and another one by LLAGNs and BHBs (see Kadowaki et al. 2015, Singh, de Gouveia Dal Pino \& Kadowaki 2015). The association between blazars and GRBs is already expected as their non-thermal radiation is commonly attributed to Doppler boosted emission by accelerated particles along the relativistic jets (as discussed in Section 2). The lack of correlation of these sources with non-blazars and BHBs in this diagram suggests that another location and/or population of relativistic particles may be producing the $\gamma$-ray emission. de Gouveia Dal Pino \& Lazarian (2005) proposed an emission model based on particle acceleration by magnetic reconnection in the core region of $\mathrm{BH}$ sources. This model was revisited more recently (Kadowaki et al. 2015; Singh et al. 2015) and a sketch of it is depicted in Figure 2 (left). There is direct evidence of this process from GRMHD simulations of accretion disks around BHs, as indicated by Figure 2 (right). Fast magnetic reconnection between the lines of the $\mathrm{BH}$ magnetosphere and those arising from the accretion flow (with rates around $0.1 V_{A}$; see Kadowaki, de Gouveia Dal Pino \& Stone in prep.) allows for the release of magnetic energy that heats and accelerates the plasma. The magnetic reconnection power calculated analytically from this core model matches quite well with the observed $\gamma$-ray luminosity of the non-blazar LLAGNs and BHBs 
plotted in the Luminosity-BH mass diagram described above (see Kadowaki et al. 2015; Singh et al. 2015)! This suggests that magnetic reconnection in the core region of these sources can explain the observed $\gamma$-ray emission. Detailed calculations of the observed spectral energy distribution (SED) of a number of BHBs (Khiali, de Gouveia Dal Pino, \& del Valle 2015) and LLAGNs, like Per A (Ramirez-Rodriguez et al. these Procs.) using this model, have demonstrated that $\mathrm{TeV}$ emission in these sources (due to proton-proton and proton-photon interactions) can be effectively reproduced by accelerated cosmic rays by reconnection, and the emission is not entirely absorbed by electron-positron pairs, as suspected before (Ramirez-Rodriguez, de Gouveia Dal Pino \& Alves-Batista, in prep.). Besides, the same process leads to neutrino emission too that can account for the observed extragalactic diffuse emission by the IceCube (see Khiali \& de Gouveia Dal Pino 2016).

\section{Summary and Conclusions}

In this talk, we have stressed the importance of turbulent magnetic reconnection and particle acceleration by reconnection in the surrounds of BHs and relativistic jets based on arguments sustained by theory and numerical simulations. Our main conclusions can be summarized as follows:

- Magnetic reconnection is important in accretion and jet systems for dissipation of magnetic energy, conversion into kinetic energy, and particle acceleration.

- In magnetized plasmas particles can be accelerated by fast magnetic reconnection (driven by turbulence) in a Fermi process and develop a power-law spectrum $N(E) \sim$ $E^{-2,-1}$

- Magnetic reconnection acceleration can be very efficient in blazar jets and produce cosmic rays with energies up to $10^{17,19} \mathrm{eV}$. These could be responsible for $\gamma$-ray flares and neutrino emission in the magnetically dominated regions of these jets. This could be also the dominating mechanism responsible for the recent observed $\gamma$-ray and neutrino emission in the TXS 0506+056 blazar and deserves further testing.

- The magnetic reconnection power can also explain the $\gamma$-ray emission from BHBs and non-blazar LLAGNs as coming from the core of these sources. This power matches well with the observed correlation of $\gamma$-ray luminosity versus $\mathrm{BH}$ mass for these sources that spans 10 orders of magnitude. Accelerated CRs by magnetic reconnection in the surrounds of these sources can produce $\mathrm{TeV} \gamma$-rays that are not entirely absorbed by pair production, as well as neutrinos (see also Ramirez-Rodriguez et al. these Procs.).

\section{Acknowledgments}

We acknowledge support from the Brazilian agencies FAPESP (2013/10559-5 grant)

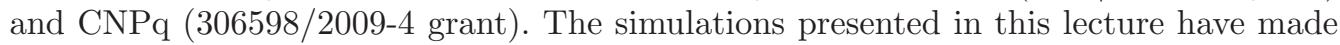
use of the computing facilities of the GAPAE group (IAG-USP) and the Laboratory of Astroinformatics IAG/USP, NAT/Unicsul (FAPESP grant 2009/54006-4).

\section{References}

Aharonian, F., Akhperjanian, A. G., Bazer-Bachi, A. R., et al. 2007, ApJ Letts., 664, L71

Clausen-Brown, E. \& Lyutikov, M. 2012, MNRAS, 426, 1374

Cerutti, B., Uzdensky, D. A. \& Begelman, M. C. 2014, ApJ, 746, 148

de Gouveia Dal Pino, E. M., \& Lazarian, A. 2005, A\&A, 441, 845

de Gouveia Dal Pino, E. M., Piovezan, P. P., \& Kadowaki, L. H. S. 2010, A\& A, 518, A5

de Gouveia Dal Pino, E. M., \& Kowal, G. 2015, in Magnetic Fields in Diffuse Media, Astrophysics and Space Science Library, Eds. A. Lazarian. E. de Gouveia Dal Pino, C. Melioli, 407, 373 de Gouveia Dal Pino, E., del Valle, M. V., Kadowaki, L., et al. 2016, Frontier Research in Astrophysics II, id.56, 56

del Valle, M. V., de Gouveia Dal Pino, E.M., \& Kowal, G. 2016, MNRAS 
Drake, J. F., Swisdak, M., Che, H., \& Shay, M. A. 2006, Nature, 443, 553

Giannios, D., Uzdensky, D. A., \& Begelman, M. C., 2009, MNRAS, 228, 395 L29-L33

Guo, F., Li, H., Daughton, W. \& Liu, Y. H. 2016, ApJ, Physics of Plasmas, 23, 055708

IceCube, T., Fermi-LAT, MAGIC, et al. 2018, Science, arXiv:1807.08816

Kadowaki, L. H. S., de Gouveia Dal Pino, E. M., \& Singh, C. B. 2015, ApJ, 802, 113

Kadowaki, L. H. S., de Gouveia Dal Pino, E. M., \& Stone, J. 2018, ApJ (in press), 2018, arXiv: $180308557 \mathrm{~K}$

Khiali, B., de Gouveia Dal Pino, E. M., \& del Valle, M. V. 2015, MNRAS, 449, 34

Khiali, B. \& de Gouveia Dal Pino, E. M. 2016, MNRAS, 455, 838

Kowal, G., Lazarian, A., Vishniac, E. T., \& Otmianowska-Mazur, K., 2009, ApJ, 700, 63

Kowal, G., de Gouveia Dal Pino, E. M., \& Lazarian, A. 2011, ApJ, 735, 102

Kowal, G., de Gouveia Dal Pino, E. M., \& Lazarian, A. 2012, PRL, 108, 241102

Kushwaha, P., Sinha, A., Misra, R., Singh, K. P., \& de Gouveia Dal Pino, E. M. 2017, ApJ, 849,138

Lazarian, A. \& Vishniac, E. T. 1999, ApJ, 517, 700

Lyubarsky, Y. \& Liverts, M. 2008, ApJ, 682, 1436

Singh, C. B., de Gouveia Dal Pino, E. M., \& Kadowaki, L. H. S. 2015, ApJ Letts., 799, L20

Singh, C. B., Mizuno, Y., \& de Gouveia Dal Pino, E. M. 2016, ApJ, 824, 48

Sironi, L., \& Spitkovsky, A. 2014, ApJ Letts., 783, L21

Sol, H., Zech, A., Boisson, C. et al. 2013, Astroparticle Physics, 43, 215

Zhang, B., \& Yan, H. 2011, ApJ, 726, 90

Zenitani, S. \& Hoshino, M. 2008, AJ, 677, 530 